\title{
ChemComm
}

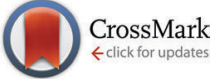

Cite this: Chem. Commun., 2015, 51,1830

Received 18th November 2014, Accepted 11th December 2014

DOI: $10.1039 / c 4 c c 09204 j$

www.rsc.org/chemcomm

\section{Switching adhesion and friction by light using photosensitive guest-host interactions}

\author{
Johanna Blass, ${ }^{\text {ab }}$ Bianca L. Bozna, ${ }^{a}$ Marcel Albrecht, ${ }^{c}$ Jennifer A. Krings, ${ }^{d}$ \\ Bart Jan Ravoo, ' Gerhard Wenz ${ }^{c}$ and Roland Bennewitz*ab
}

\begin{abstract}
Friction and adhesion between two $\beta$-cyclodextrin functionalized surfaces can be switched reversibly by external light stimuli. The interaction between the cyclodextrin molecules attached to the tip of an atomic force microscope and a silicon wafer surface is mediated by complexation of ditopic azobenzene guest molecules. At the single molecule level, the rupture force of an individual complex is $61 \pm 10 \mathrm{pN}$.
\end{abstract}

Surfaces with switchable adhesion or friction characteristics present a new field of research and promise great potential in the field of adhesives or tribological systems controlled by external stimuli. Self-assembled monolayers are one promising approach to provide surfaces with switchable functions, examples being the switching of wettability ${ }^{1}$ or of nanomechanical properties. $^{2}$ In this field, azobenzene-derived systems are of special interest as azobenzene has been shown to change its configuration instantly but reversibly by irradiation with ultraviolet (UV) and visible (VIS) light, respectively, ${ }^{3}$ without generating any side products. ${ }^{4}$ Thus, azobenzenes have been used in supramolecular assemblies, ${ }^{5}$ in covalent functionalization of metal surfaces, ${ }^{4}$ or for the switching of bioactivity by controlling the binding of azobenzene modified glycoconjugates to cyclodextrin monolayers. ${ }^{6}$ Atomic force microscopy (AFM) allows to study mechanical properties of surface-linked azobenzene-derivatives at the single molecule level. ${ }^{7}$

In this letter, we present a novel surface functionalization based on cyclodextrin assemblies with photo switchable friction and adhesion characteristics mediated by ditopic azobenzene connector molecules in solution. Azobenzene moieties can be isomerized reversibly from trans to cis states by irradiation with

\footnotetext{
${ }^{a}$ INM - Leibniz-Institute for New Materials, Saarland University, Campus D2 2, 66123 Saarbrücken, Germany. E-mail: roland.bennewitz@inm-gmbh.de

${ }^{b}$ Physics Department, Saarland University, Saarland University, Campus D2 2, 66123 Saarbrücken, Germany

' Organic Macromolecular Chemistry, Saarland University, Campus C4 2, 66123 Saarbrücken, Germany

${ }^{d}$ Organic Chemistry Institute, University of Münster, Corrensstrasse 10, 48149 Münster, Germany
}

light at $365 \mathrm{~nm}$ and from cis to trans at $455 \mathrm{~nm}$. The rod like trans state of the azobenzene is apolar and binds efficiently into the cyclodextrin cavities whereas the bent cis form is much more polar and sterically hindered from complexation. ${ }^{8}$ Force measurements by AFM are sensitive enough to detect single host-guest interactions. These interactions have been studied in detail for various guests in cyclodextrin (CD) hosts. ${ }^{9}$

We use AFM to study the molecular mechanisms underlying friction and adhesion. A $\beta$-cyclodextrin ( $\beta$-CD) monolayer is attached to both the oxidized silicon tip of the AFM and the oxidized silicon wafer substrate, see the scheme in Fig. 1(a). The attachment is realized by first tethering a silane adlayer with isothiocyanate groups in tetrahydrofuran to the silicon oxide surfaces. The $\beta$-CD molecules carry an amine functionality ${ }^{10}$ which is able to form a stable thiourea bond by reaction in

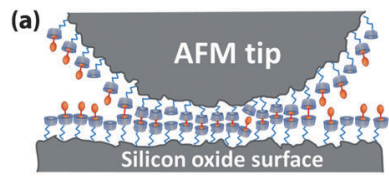

(b)

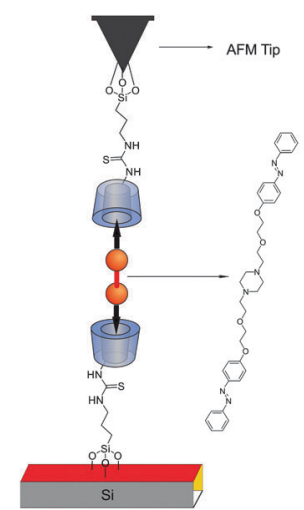

Fig. 1 (a) Schematic representation of the experiment. Tip and surface are functionalized by $\beta$-cyclodextrin molecules. Both end groups of ditopic guest connector molecules form complexes with $\beta$-cyclodextrin molecules at tip and surface, resulting in adhesion and friction. (b) Molecular structures of the compounds. 
water with the isothiocyanate groups at the surfaces. Attractive interactions between the $\beta$-CD molecules at tip and surface arise from complexation of ditopic guest connector molecules, see Fig. 1(b). The synthesis of the ditopic guest molecules and their photo-responsive complexation with cyclodextrin molecules has been described elsewhere. ${ }^{8}$ Typical association constants for trans-azobenzene derivatives with $\beta$-CD are of the order of $2.5 \times 10^{3} \mathrm{M}^{-1},{ }^{11}$ while the binding affinity of cis-azobenzene is too small for quantification. For the binding of azobenzene in $\beta$-CD monolayers an association constant of $5.8 \times 10^{3} \mathrm{M}^{-1}$ has been reported. ${ }^{6}$ The ditopic connector molecules carry two hydrophobic end groups which form a specific non-covalent bond between two opposing $\beta$-CD cavities via hydrophobic interactions.

All force microscopy experiments were performed at room temperature, using a JPK NanoWizard3 AFM and a rectangular silicon cantilever with a nominal force constant of $0.2 \mathrm{~N} \mathrm{~m}^{-1}$. Normal force measurements were calibrated following the methods introduced by Sader and co-workers. ${ }^{12}$ Lateral force measurements were calibrated using the wedge method in air $^{13}$ (sample TGG01 from Micromash, Sofia, Bulgaria) and applying the correction factor for experiments in liquid introduced by Tocha et al. ${ }^{14}$

A typical example for a force-distance curve is shown in Fig. 2(a). The force between tip and surface is recorded while the AFM cantilever is approached to and retracted from the surface. Two stepwise increases of the attractive force are observed when the tip apex approaches the surface, indicating a molecular snap-into-contact through complexation events. The rupture of
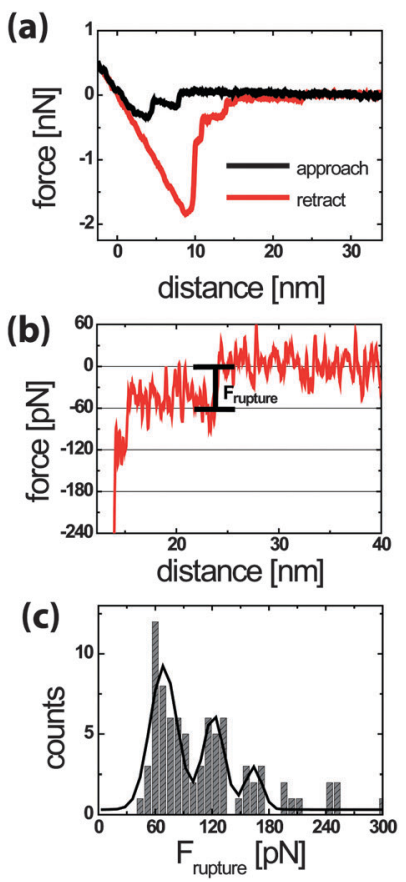

Fig. 2 (a) Representative force-distance curve recorded in the guest connector solution. Formation of non-covalent bonds can be observed upon approach, a high pull-off force and the subsequent rupture of multiple complexes are observed during retraction. (b) Detail of the retract curve in (a), showing the last rupture event and the related force drop $F_{\text {rupture. }}(\mathrm{c})$ Histogram showing the distribution of $F_{\text {rupture. The solid line }}$ represents the sum of Gaussian curves fitted to the peak.
$\beta$-CD-azobenzene complexes occurs in multiple steps during the retraction of the AFM cantilever from the surface. Each force drop reflects the rupture of multiple or single complexes. To determine the characteristic rupture force for an individual complex, the distribution of the last force drops, labelled $F_{\text {rupture }}$ in Fig. 2(b), is plotted as a histogram in Fig. 2(c). Only the last rupture event of each curve is considered to ensure that the cantilever is fully relaxed after the rupture event. The histogram exhibits multiple maxima with equidistant spacing corresponding to the rupture of a single complex, parallel rupture of two complexes etc. The maxima lie at multiples of $61 \pm 10 \mathrm{pN}$, which is the characteristic rupture force for a single complex. In an ongoing study of ditopic guest connector molecules with adamantane end groups we have found a single complex rupture force of $82 \pm 10 \mathrm{pN}$. The lower rupture force for the azobenzene connector is consistent with the lower binding constant for complexes of azo derivatives with $\beta$-CD of $K=2.5 \times 10^{3} \mathrm{M}^{-1}$ (ref. 11) as compared to the one for adamantane derivatives with $\beta$-CD of $K=4.6 \times 10^{4} \mathrm{M}^{-1} .^{15}$

Previous force microscopy studies with various monotopic guests have reported rupture forces ranging from 39 to $102 \mathrm{pN}$, where adamantane guests form the strongest complexes with cyclodextrin molecules. ${ }^{9 a}$ The maximum of the histogram indicates the most probable force to rupture one of two complexes connected in series, while the most probable force to rupture one single azobenzene- $\beta$-CD complex would probably be a little higher.

The influence of the connector molecules and of their lightinduced isomerization on the maximum pull-off force $(1.8 \mathrm{nN}$ in the example in Fig. 2(a)) is summarized in Fig. 3. Control experiments were performed in ultrapure water. Connector molecules were diluted in dimethyl sulfoxide (DMSO) and added to the ultrapure water in a ratio of $1: 10$, resulting in $10 \mu \mathrm{M}$ concentration.
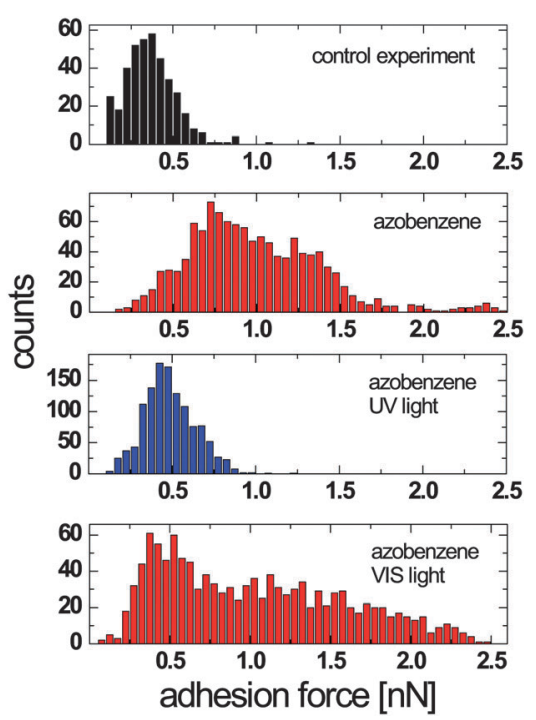

Fig. 3 Histograms of pull-off forces recorded in a series of experiments for a $\beta$-CD functionalized AFM-tip and surface in a control experiment in pure water, in a solution of azobenzene connector molecules, after irradiation with UV light (367 nm), and after irradiation with VIS (454 nm) light. 
Each histogram reflects the distribution of pull-forces from 1200 force-distance curves (pulling rate $1 \mu \mathrm{m} \mathrm{s}^{-1}$, maximum load $0.5 \mathrm{nN}$ ) recorded in 70 minutes. The histograms show the significant effect of the ditopic connector molecules on the adhesion force.

In the control experiment, non-specific tip-sample interactions result in a narrow distribution of pull-off forces around $0.35 \mathrm{nN}$. After adding the guest molecules the spectrum of adhesion forces becomes much wider with pull-off forces between 0.5 and $1.5 \mathrm{nN}$, some even up to $2.5 \mathrm{nN}$. Irradiation with the light of a UV LED (peak intensity at $367 \mathrm{~nm}, 10.5 \mathrm{~nm}$ width) results in a decrease of pull-off forces to the values of control experiment. By subsequent irradiation with VIS light (broad-spectrum white light LED with peak intensities at $454 \mathrm{~nm}$ and $553 \mathrm{~nm}$ ) the system was switched back to the high adhesion state with pull-off forces ranging again from 0.3 to $2.5 \mathrm{nN}$. While the low pull-off force values around $0.3 \mathrm{nN}$ observed in the control experiment are not observed after initially adding the connector molecules, they are observed after the sequence of UV and VIS irradiation. The transition of azobenzene end groups from the cis to the trans state under VIS irradiation may not be complete and, therefore, non-specific interactions may arise from competition of ditopic complex formation with only partially isomerized connector molecules.

Only a low concentration of guest molecules in the waterDMSO solution $(10 \mu \mathrm{M})$ is required to achieve these significant effects on adhesion. About 60 complexes contribute to adhesion. This rough upper bound estimate can be made by taking the product of the maximum packing density of $\beta$-CD molecules of $0.33 \mathrm{~nm}^{-2}$ and an estimated contact area derived from the tip radius of $200 \mathrm{~nm}^{2}$ and by assuming that most $\beta$-CD molecules are complexed. While changing the light stimuli, the AFM-tip was retracted about $3 \mu \mathrm{m}$ from the surface, resulting in a time gap of less than five minutes between each series of force-distance curves. The results presented here were achieved with illumination of tip and sample from the side. Quantitatively similar results were obtained for a borosilicate glass substrate functionalized in the same way, where tip and sample were illuminated through the substrate.

The molecular complex formation and its switching by light stimuli also lead to a significant friction contrast. The result of a series of friction force microscopy experiments is summarized in Fig. 4. The friction force is determined from the torsional bending of the AFM cantilever when the tip slides in contact with the surface. ${ }^{16}$ In this configuration, friction can be switched reversibly by a factor of more than two by irradiation with UV and VIS light. The friction force decreases upon UV irradiation almost to the value of the control experiment without azobenzene connector molecules. The measurements were performed by scanning lines of $50 \mathrm{~nm}$ back and forth, i.e. several times the estimated contact diameter, in 1 second. The normal load was kept at a low value of $0.5 \mathrm{nN}$ in order to minimize non-specific and deformation contributions to the friction signal. The AFM tip was retracted for 1 minute while switching the irradiation wavelength. Typical lateral force traces are shown in Fig. 4(b) for a control in pure water and for irradiation with UV and VIS light in the guest molecule solution. The complex formation by the guest connector molecules under VIS irradiation does not (a)

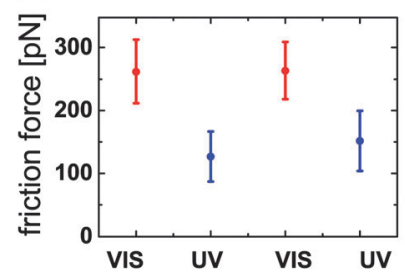

(b)

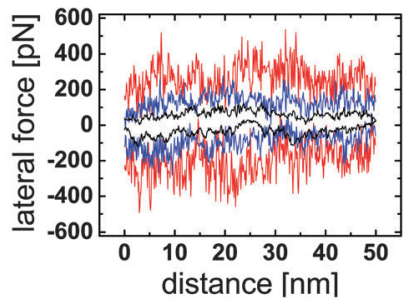

Fig. 4 (a) Light-responsive friction between $\beta$-CD functionalized AFM-tip and silicon surface in azobenzene connector molecule solution. The friction force is calculated as the average lateral force during one forward and one backward scan over $50 \mathrm{~nm}$. The error bars represent the standard deviation of 512 scan lines. (b) Typical lateral force traces for forward and backward scan in pure water as control experiment (black line) and in azobenzene connector molecule solution during irradiation with VIS light (red) and UV light (blue).

only cause higher average friction, but also changes the characteristic appearance of the lateral force traces.

While the non-specific interactions in the control experiments lead to a smooth lateral force curve with some minor fluctuations, the rupture and rebinding of guest-host complexes produces irregular stick-slip motion of the AFM tip which is revealed as saw-tooth characteristic of the lateral force. Typical force drops in the stick-slip force trace have values scattering around $250 \mathrm{pN}$, comparable to the force drops observed in the force-distance curves in Fig. 2(a) when multiple non-covalent bonds were broken. The friction process can thus be described in the following way. The AFM tip is stuck in place by multiple non-covalent bonds, while the lateral force is built up by the pulling of the cantilever. When the lateral force reaches a threshold value, one or usually multiple bonds are broken and the tip slips to a new position where a different configuration of non-covalent bonds leads to a further sticking phase. This process is continuously repeated, as each new position of the tip leads to rebinding with new $\beta$-CD molecules at the surface. The force trace recorded in guest molecule solution under UV irradiation reveals significantly lower average friction than that recorded under VIS irradiation, but exhibits some signatures of the stick-slip characteristic, reflecting that even under UV irradiation a fraction of about $20 \%$ of the connector molecules is still in the trans-state and contribute to the molecular binding process. ${ }^{17}$

In conclusion, we present a novel surface functionalization of host monolayers with switchable friction and adhesion properties mediated by photoresponsive connector molecules. At the molecular level, the rupture force of an individual hostguest complex formed by $\beta$-cyclodextrin molecules and the two azobenzene end groups of the connector molecule is $61 \pm 10 \mathrm{pN}$. The pull-off force between functionalized AFM tip and silicon surface can be switched by a factor of five by irradiation with UV and VIS light, similarly the friction can be switched by a factor of two. The novel surface functionalization is a first example to demonstrate active control of adhesion and friction by means of macromolecular guest-host chemistry. The molecular design of our approach allows for different guest connector molecules binding the same surface functionalization, offering a great variability in the molecular control of friction and adhesion. 
The authors thank the Volkswagen Foundation for financial support through the program Integration of Molecular Components in Functional Macroscopic Systems. Furthermore, Devid Hero is gratefully acknowledged for his synthetic support in the preparation of some cyclodextrin derivatives.

\section{Notes and references}

1 J. Lahann, S. Mitragotri, T. N. Tran, H. Kaido, J. Sundaram, I. S. Choi, S. Hoffer, G. A. Somorjai and R. Langer, Science, 2003, 299, 371.

2 (a) Y. F. Dufrêne, W. R. Barger, J. B. D. Green and G. U. Lee, Langmuir, 1996, 13, 4779; (b) D. J. Díaz, J. E. Hudson, G. D. Storrier, H. D. Abruña, N. Sundararajan and C. K. Ober, Langmuir, 2001, 17, 5932; (c) M. Lemieux, D. Usov, S. Minko, M. Stamm, H. Shulha and V. V. Tsukruk, Macromolecules, 2003, 36, 7244; (d) V. Kolivoška, M. Mohos, I. V. Pobelov, S. Rohrbach, K. Yoshida, W. J. Hong, Y. C. Fu, P. Moreno-Garcia, G. Mészáros, P. Broekmann, M. Hromadová, R. Sokolová, M. Valàšek and T. Wandlowski, Chem. Commun., 2014, 50, 11757.

3 (a) T. Kobayashi, E. O. Degenkolb and P. M. Rentzepis, J. Phys. Chem., 1979, 83, 2431; (b) I. K. Lednev, T. Q. Ye, R. E. Hester and J. N. Moore, J. Phys. Chem., 1996, 100, 13338; (c) W. R. Browne and B. L. Feringa, Annu. Rev. Phys. Chem., 2009, 60, 407.

4 K. G. Yager and C. J. Barrett, J. Photochem. Photobiol., A, 2006, 182, 250.

5 Z. Liu and M. Jiang, J. Mater. Chem., 2007, 17, 4249.

6 J. Voskuhl, S. Sankaran and P. Jonkheijm, Chem. Commun., 2014, 50, 15144.
7 N. B. Holland, T. Hugel, G. Neuert, A. Cattani-Scholz, C. Renner, D. Oesterhelt, L. Moroder, M. Seitz and H. E. Gaub, Macromolecules, 2003, 36, 2015.

8 S. K. M. Nalluri and B. J. Ravoo, Angew. Chem., Int. Ed., 2010, 49, 5371.

9 (a) H. Schönherr, M. W. J. Beulen, J. Bugler, J. Huskens, F. van Veggel, D. N. Reinhoudt and G. J. Vancso, J. Am. Chem. Soc., 2000, 122, 4963; (b) S. Zapotoczny, T. Auletta, M. R. de Jong, H. Schonherr, J. Huskens, F. van Veggel, D. N. Reinhoudt and G. J. Vancso, Langmuir, 2002, 18, 6988; (c) T. Auletta, M. R. de Jong, A. Mulder, F. van Veggel, J. Huskens, D. N. Reinhoudt, S. Zou, S. Zapotoczny, H. Schönherr, G. J. Vancso and L. Kuipers, J. Am. Chem. Soc., 2004, 126, 1577; (d) A. Gomez-Casado, H. H. Dam, M. D. Yilmaz, D. Florea, P. Jonkheijm and J. Huskens, J. Am. Chem. Soc., 2011, 133, 10849; (e) O. Kaftan, S. Tumbiolo, F. Dubreuil, R. Auzely-Velty, A. Fery and G. Papastavrou, J. Phys. Chem. B, 2011, 115, 7726.

10 G. Wenz, C. Strassnig, C. Thiele, A. Engelke, B. Morgenstern and K. Hegetschweiler, Chem. - Eur. J., 2008, 14, 7202.

11 A. Samanta and B. J. Ravoo, Chem. - Eur. J., 2014, 20, 4966.

12 C. Green, H. Lioe, J. Cleveland, R. Proksch, P. Mulvaney and J. Sader, Rev. Sci. Instrum., 2004, 75, 1988.

13 H. Wang and L. M. Gee, Ultramicroscopy, 2014, 136, 193.

14 E. Tocha, J. Song, H. Schönherr and G. J. Vancso, Langmuir, 2007, 23, 7078 .

15 A. Mulder, T. Auletta, A. Sartori, S. Del Ciotto, A. Casnati, R. Ungaro, J. Huskens and D. N. Reinhoudt, J. Am. Chem. Soc., 2004, 126, 6627.

16 E. Gnecco, R. Bennewitz, T. Gyalog and E. Meyer, J. Phys.: Condens. Matter, 2001, 13, R619.

17 S. K. M. Nalluri, J. Voskuhl, J. B. Bultema, E. J. Boekema and B. J. Ravoo, Angew. Chem., 2011, 123, 9921. 